\title{
Percepção de docentes de Odontologia sobre o uso de dentes humanos como recurso educativo em atividades pré-clínicas
}

\author{
Iago Bruno da Silva*; Tâmara Tiffany Ferreira*; Elias Gomes Ferreira da Silva*; Andressa Barcelos \\ Aires Barros*; Lila Louise Moreira Martins Franco**; Brunno Santos de Freitas Silva**; Raquel \\ Baroni de Carvalho***; Leandro Brambilla Martorell** \\ * Egresso(a), Curso de Odontologia, Centro Universitário de \\ Anápolis, UniEvangélica \\ ** Docente, Curso de Odontologia, Centro Universitário de \\ Anápolis, UniEvangélica \\ *** Docente, Curso de Odontologia, Universidade Federal do \\ Espírito Santo
}

Recebido em 30/10/2019. Aprovado em 21/06/2020.

\begin{abstract}
RESUMO
O estudo teve por objetivo avaliar a percepção de docentes de Odontologia em relação à utilização de dentes humanos extraídos e dentes artificiais em atividades pré-clínicas. Utilizou-se um questionário com perguntas objetivas e subjetivas aplicado aos professores cirurgiões-dentistas do Curso de Odontologia Centro Universitário de Anápolis - UniEVANGÉLICA. Dos 50 professores que correspondiam aos critérios de elegibilidade, obteve-se taxa de resposta de $76 \%$. A média do tempo de formado dos docentes é de $20 \pm 9,7$ anos e a média do tempo de docência de $17 \pm 10,5$ anos. Quando questionados se dentes humanos deveriam ser utilizados como recursos educativos, a maioria $(89,4 \%)$ respondeu que sim e em relação à aquisição de habilidades psicomotoras dos estudantes $68,4 \%$ acreditam que os dentes humanos são mais vantajosos. Citaram como desvantagem do uso aspectos relacionados à biossegurança $(36,8 \%)$, dificuldade para obtenção dos dentes $(21 \%)$, riscos de comercialização $(15,7 \%)$ e aspectos éticos $(15,7 \%)$. A maioria dos docentes de Odontologia do curso avaliado entendem que o uso de dentes humanos em atividades pré-clínicas tem maior potencial pedagógico, além de favorecer o desenvolvimento de habilidades motoras em comparação ao uso de dentes artificiais.
\end{abstract}

Descritores: Ensino Odontológico. Dente. Docentes de Odontologia.

\section{INTRODUÇÃO}

As Diretrizes Curriculares Nacionais para os cursos de graduação em Odontologia (DCN) publicadas em $2002^{1}$, assim como o parecer de sua atualização $(2018)^{2}$, elencam os conteúdos que devem ser abordados de forma integrada durante a formação do estudante: ciências biológicas e da saúde; ciências humanas e sociais; e ciências 
odontológicas.

Dentre os componentes curriculares que trabalham com os conteúdos das ciências odontológicas existem aqueles que buscam o aprimoramento de habilidades psicomotoras para a execução de procedimentos clínicos. Todavia, antes do treinamento com pacientes, recomenda-se a realização de treinamentos pré-clínicos ${ }^{3}$. Tradicionalmente, as atividades pré-clínicas utilizam dentes humanos extraídos, entretanto, atualmente são utilizados igualmente ${ }^{4}$ os dentes artificiais, de resina opaca ou transparente ${ }^{5}$, ou até mesmo blocos de resina ${ }^{6}$.

A literatura tem apontado vantagens e desvantagens no uso destes diferentes modelos. Sobre o uso de dentes humanos destacam-se a necessidade de procedimentos como desinfecção e esterilização ${ }^{7}$ pelo risco de infecção cruzada; a variabilidade anatômica, o que dificulta a avaliação do desempenho individual de um estudante; e até mesmo a dificuldade de obtenção do dente ${ }^{8}$, o que evidencia também um dilema moral e legal ${ }^{9}$ para a prática, que pode favorecer atos ilegais de comercialização de dentes humanos ${ }^{10}$, conforme relatado por estudantes de graduação em pesquisa recente sobre o tema ${ }^{11}$.

Já os dentes artificiais não trazem risco de infecção, estão disponíveis em grande número, são uniformes o que permite uma avaliação validada, além de permitir aos estudantes a prática do mesmo procedimento até obter resultados consistentemente bons com uma dada técnica ou para um dado desafio anatômico ${ }^{5,8}$. Por outro lado, são apontadas como falhas a diferença entre a dureza da resina em comparação com o dente ${ }^{12} \mathrm{e}$ diferença de viscosidade do material da câmara pulpar e do canal radicular, o que o torna de difícil remoção para as práticas de endodontia ${ }^{5}$.

A despeito destas diferenças entre dentes humanos extraídos e dentes artificiais, pesquisas têm buscado evidenciar a percepção de professores $^{5}$ e estudantes ${ }^{12}$ em relação às diferenças entre estes dois grupos, e se é possível substituir o uso de dentes humanos por dentes artificiais, comparando o desempenho de estudantes durante as atividades pré-clínicas ${ }^{8,13}$. Esta pesquisa investigou a percepção dos docentes do Curso de Odontologia do Centro Universitário de Anápolis - UniEVANGÉLICA sobre o uso de dentes humanos em relação aos artificiais em atividades pré-clínicas.

\section{METODOLOGIA}

Esta pesquisa foi aprovada pelo Comitê de Ética em Pesquisa do Centro Universitário de Anápolis - Unievangélica (CAEE 80561317. 8.0000.5076). A população do estudo consiste em 67 docentes. Entretanto, foram incluídos na amostra apenas os docentes com formação em Odontologia e excluídos aqueles envolvidos diretamente e autores do presente estudo, resultando em 50 professores elegíveis. A coleta de dados aconteceu por meio de aplicação de questionário enviado pela plataforma do GoogleForms ${ }^{\circledR}$, o qual foi reenviado duas vezes, com intervalo de 10 dias. Após este prazo os docentes não respondentes foram contatados presencialmente, oportunizando a resposta em questionário impresso.

O questionário (figura 1), elaborado pelos próprios pesquisadores, é composto por 12 perguntas, abertas e fechadas e a análise dos dados foi realizada por meio de frequências relativa e absoluta para as perguntas fechadas, e por meio de análise de conteúdo ${ }^{14}$, com criação de categorias, para as perguntas abertas.

\section{RESULTADOS}

Dos 50 professores elegíveis para o estudo, 38 responderam aos questionários, encontrando-se uma taxa de resposta de $76 \%$. A média do tempo de formado dos docentes é de 20 anos $( \pm 9,7)$ e a média do tempo de docência de 17 anos $( \pm 10,5)$.

Apenas um $(2,6 \%)$ professor afirmou não ter 


\section{Aspectos Gerais}

1. Em que ano o(a) senhor(a) concluiu a graduação?

2. Atualmente, qual a sua área de atuação no curso de Odontologia?

3. Há quanto tempo está na docência nesta(s) área(s) (especificar cada uma)?

\section{Utilização de dentes como recurso educativo}

4. Durante a sua graduação você utilizou dentes humanos extraídos em atividades pré-clínicas?
( ) Sim ( ) Não ( ) Não me lembro

5. Em quais áreas? Pode assinalar mais de uma se for o caso.

( ) Anatomia e escultura dental ( ) Dentística ( ) Endodontia ( ) Prótese ( ) Outras:

6. Como você teve acesso a estes dentes (especificar para cada uma das áreas)?

7. Você considera que dentes humanos extraídos devem ser usados em prática laboratorial como recurso educativo?

( ) Sim ( ) Não ( ) Não sei Por favor, justifique sua resposta.

8. Há vantagens para o uso de dentes humanos extraídos em prática laboratorial?

Quais?

9. Há desvantagens para o uso de dentes humanos extraídos em prática laboratorial?

Quais?

10. No que tange ao potencial didático do recurso educativo, qual tipo de dentes garantiria uma prática laboratorial mais vantajosa?

( ) Dentes artificiais ( ) Dentes humanos ( ) Os dois igualmente Qual tipo de potencial didático está relacionado com a sua resposta?

11. No que tange ao desenvolvimento de habilidades psicomotoras por parte dos estudantes, qual tipo de dentes garantiria uma prática laboratorial mais vantajosa?

( ) Dentes artificiais ( ) Dentes humanos ( ) Os dois igualmente Por quê?

12. Quais são as implicações éticas e legais do uso de dentes humanos em práticas pré-clínicas nos cursos de Odontologia?

Figura 1. Questionário aplicado aos docentes

usado dentes humanos em sua formação na graduação, todos os $37(97,4 \%)$ restantes declaram ter utilizado dentes em disciplinas de Endodontia, $26(66,6 \%)$ em disciplinas de Dentística; 22 $(56,4 \%)$ em Anatomia e Escultura Dental; 9 (23\%) em Prótese; e $1(2,6 \%)$ tanto em Cirurgia quanto em Radiologia.

Sobre a origem dos dentes que utilizaram observou-se a indicação de cirurgiões-dentistas de clínicas particulares por 19 (50\%); serviços públicos de saúde ("postos de saúde" "hospital municipal") por $11(28,9 \%)$; colegas de graduação por $4(10,5 \%)$; e clínicas da própria faculdade por
$2(5,2 \%)$. Apenas $6(15,7 \%)$ afirmaram que a disciplina ofertava os dentes, sendo $1(2,6 \%)$ por meio do Banco de Dentes Humanos (BDH). Em geral, quando os dentes eram fornecidos tratava-se da disciplina de Anatomia e Escultura Dental. Ainda, 2 (5,2\%) declararam terem comprado dentes e $2(5,2 \%)$ não responderam.

Quando questionados se dentes humanos deveriam ser utilizados como recursos educativos, a maioria (34-89,4\%) respondeu que sim, $3(7,8 \%)$ que não e $1(2,6 \%)$ disse não saber. Para os que concordam com o uso foi possível perceber as categorias "realismo", "utilidade" e "aspectos 
éticos". $\mathrm{Na}$ primeira categoria $28 \quad(73,6 \%)$ destacaram as características anatômicas da coroa, raiz e condutos radiculares, a dureza do esmalte $\mathrm{e}$ dentina que permitem uma simulação mais próxima das características que os estudantes encontrarão na clínica. Na categoria "utilidade" 2 $(5,2 \%)$ destacaram que regra geral dentes extraídos são descartados, assim, poderiam ser utilizados para o ensino. Já na categoria "aspectos éticos" 4 $(10,5 \%)$ destacaram que o uso só deveria ser feito mediante ao respeito das normas éticas. Para os que não concordam a categoria utilizada foi a de "modelos alternativos", sendo citadas resinas com dureza próxima à da dentina e também a questão do melhor uso de dentes para armazenamento de células-tronco.

Os professores citaram como desvantagem do uso aspectos relacionados à biossegurança (14 $36,8 \%$ ), dificuldade para obtenção dos dentes (8 $21 \%$ ), riscos de comercialização (6 $-15,7 \%)$, e aspectos éticos $(6-15,7 \%)$. Ainda foram citadas como desvantagens a banalização do órgão, podendo estimular a ideia de que dentes são menos importantes, o mau cheiro causado pelo uso da broca no dente humano em atividade laboratorial e características do dente acessado (cor, presença de cárie, etc.).

Quando questionados em relação ao potencial didático do recurso de treinamento 23 $(60,5 \%)$ acreditam que os dentes humanos são mais vantajosos, $4(10,5 \%)$ preferem os dentes artificiais e $11(28,9 \%)$ consideram ambos igualmente. Sobre a percepção dos professores em relação à aquisição de habilidades psicomotoras dos estudantes, 26 $(68,4 \%)$ acreditam que os dentes humanos são mais vantajosos, $1(2,6 \%)$ os dentes artificiais e 11 $(28,9 \%)$ consideram os dois igualmente.

Por fim, quando questionados sobre as implicações éticas e legais do uso de dentes humanos, $2(5,2 \%)$ acreditam que não existem, uma vez que são dentes extraídos e que serão descartados, $5(13,1 \%)$ não sabem ou não responderam. Apenas $8(21 \%)$ citaram a questão da necessidade do consentimento do paciente e somente $3(7,8 \%)$ a possibilidade de o uso de dentes incentivar a prática de exodontias desnecessárias. Ainda, 7 (18,4\%) citaram a comercialização de dentes humanos e $16(42,1 \%)$ deram respostas mais generalistas que apontam a necessidade de seguir regras (código de ética e leis) no uso de dentes humanos. Ainda, $7(18,4 \%)$ citaram o BDH como instituição que poderia servir na superação destes problemas.

\section{DISCUSSÃO}

A maioria dos docentes entende que os dentes humanos devem ser utilizados nas práticas pré-clínicas, sendo estes de maior potencial didático $\mathrm{e}$ os que mais oportunizam $\mathrm{o}$ desenvolvimento de habilidades psicomotoras dos estudantes, quando comparados com os dentes artificiais. Os três estudos apresentados nos parágrafos seguintes estão em acordo com o encontrado nesta pesquisa.

Um estudo realizado com 18 professores brasileiros de Endodontia buscou avaliar a anatomia e características físicas e radiográficas de dentes artificiais para o ensino na área. Aos professores de diferentes cursos foi solicitado realizarem simulações com os dentes de uma marca comercial e responderem a um questionário sobre esta experiência. As características melhor avaliadas foram a anatomia interna e externa e a localização do canal radicular. Entretanto, 33\% da amostra considerou a dureza do material insatisfatória para a prática de ensino, por dar a impressão errada de não existir muita resistência para acessar a câmara pulpar em dentes naturais ${ }^{5}$.

Outro estudo buscou comparar o tempo de preparação e as percepções de dificuldade de graduandos em Odontologia e endodontistas em realizar preparos de canais radiculares em modelos opacos de resina e dentes naturais extraídos. Para tanto foram selecionados 10 estudantes do $5^{\circ}$ ano e 
10 especialistas com mínimo de 5 anos de experiência e cada um preparou 2 dentes de cada modelo. Os dois grupos relataram problemas com a anatomia, dificuldade para irrigar e obturar o canal, bem como para interpretar as radiografias. Apesar do tempo de realização dos procedimentos em dentes humanos ter sido maior, o estudo concluiu que nenhum dos dois modelos apresentou características que permitissem a substituição de dentes humanos para o ensino ${ }^{6}$.

Em outra pesquisa, 43 estudantes foram alocados aleatoriamente em dois grupos para realizarem tratamentos endodônticos em dentes humanos e artificiais. Esta pesquisa concluiu que o controle do sucesso do treinamento de estudantes usando dentes naturais pode ser mais confiável do que quando utilizados os dentes artificiais ${ }^{13}$.

Mais recentemente, uma revisão sistemática da literatura ${ }^{15}$ buscou comparar os resultados educacionais que poderiam ser alcançados com o uso dentes artificiais versus dentes extraídos, especificamente para treinamento endodôntico préclínico. Foram incluídos apenas cinco estudos que totalizaram a avaliação de 359 operadores, sendo 349 (97\%) estudantes de graduação e 10 (3\%) endodontistas. Em relação aos resultados técnicos, não foram encontradas diferenças significativas entre o treinamento com dentes artificiais e dentes extraídos, mas o desempenho tendeu a ser melhor nos dentes artificiais. Os operadores treinados apenas em dentes artificiais pareciam ser adequadamente instruídos para o tratamento subsequente do canal radicular no contexto clínico. Com base nas evidências disponíveis os autores concluíram que o uso de dentes artificiais para treinamento endodôntico pré-clínico alcançou resultados educacionais semelhantes em comparação aos dentes extraídos. No entanto, as experiências relatadas pelos operadores divergiram e mais estudos precisam ser feitos.

Outros estudos têm apontado a utilização de tecnologias de realidade virtual para a substituição de dentes humanos ou até mesmo de modelos artificiais em atividades pré-clínicas de estudantes de odontologia. Uma pesquisa avaliou a habilidade de vinte estudantes antes e depois de seu treinamento utilizando um sistema de realidade virtual háptico de endodontia e concluíram que estes demonstraram aprender a executar tarefas de abertura e de acesso mais rapidamente e com mais consistência, com melhor destreza bimanual e melhor utilização da força ${ }^{16}$. Um recente estudo randomizado realizado com 88 estudantes do primeiro ano de graduação na França trouxe resultados semelhantes na comparação de desempenho nos grupos que treinaram em dentes de plástico e em mecanismos de realidade virtual. Em suas conclusões apontaram para as vantagens do uso da realidade virtual uma vez que traz economia de tempo de supervisão e ensino e reduz a necessidade de utilização de materiais plásticos ${ }^{17}$.

A maior desvantagem indicada pelos docentes da atual pesquisa em relação ao uso de dentes humanos se relaciona com as dificuldades de biossegurança. Esta preocupação deve ser considerada principalmente porque em regra geral, os estudantes que são responsáveis por operacionalizar o material de trabalho. Uma pesquisa foi realizada com 100 estudantes de Odontologia no Irã identificou-se que nem todos tomavam os devidos cuidados na manipulação destes dentes. Por exemplo, $87 \%$ dos entrevistados desinfetavam os dentes extraídos, $79 \%$ dos participantes usavam máscara, $84 \%$ usavam luvas e avental branco, e $61 \%$ usavam óculos de segurança enquanto trabalhavam ${ }^{18}$.

Outra situação trazida pelos docentes relaciona-se com os aspectos éticos e legais para obtenção dos dentes humanos bem como uma preocupação de exposição de pacientes vulneráveis a extrações desnecessárias caso os dentes extraídos recebam grande valorização no ensino odontológico. Sobre a comercialização, um estudo aplicando questionário com 182 estudantes 
encontrou que $11 \%$ destes já haviam comprado dentes humanos. Os dentes eram solicitados por 5 diferentes disciplinas, sendo que o número de dentes solicitados para cada estudante variou de 2 a $14^{10}$.

Além disso, é importante ressaltar que ainda que alguns docentes tenham declarado que o dente extraído pode ser utilizado sem maiores considerações por geralmente ser considerado um resíduo do serviço de saúde, é mandatório respeitar a vontade do paciente, solicitando a sua autorização para os possíveis usos 9 .

Por fim, alguns docentes mencionaram a desvantagem do uso de dentes humanos extraídos por estes permitirem o acesso às células-tronco dos indivíduos. O uso de células-tronco dentárias já demonstrou, em estudos pré-clínicos, resultados favoráveis para a regeneração óssea e no tratamento de doenças periodontais. Entretanto, estudos clínicos ainda estão sendo realizados para se conhecer a viabilidade clínica destas abordagens, tornando esta desvantagem ainda potencial, uma vez que além de provada a possibilidade do uso clínico, é preciso que a tecnologia utilizada seja acessível à população ${ }^{19}$.

\section{CONCLUSÃO}

Neste estudo foi possível concluir que a maioria dos docentes de Odontologia do curso avaliado entendem que o uso de dentes humanos em atividades pré-clínicas tem maior potencial pedagógico, além de favorecer o desenvolvimento de habilidades motoras em comparação ao o uso de dentes artificiais.

\section{ABSTRACT \\ Perception of dental professors about the use of human teeth as an educational resource in pre-clinical activities}

The present study aimed to assess the dental professor perception in relation to the use of extracted human teeth and artificial teeth in preclinical activities. A questionnaire was used with objective and subjective questions applied to professors of dental surgeons from the Dentistry Course Centro Universitário de Anápolis UniEVANGÉLICA. Of the 50 professors who met the eligibility criteria, a response rate of $76 \%$ was obtained. The average length of time for professors to graduate is $20 \pm 9.7$ years and the average teaching time is $17 \pm 10.5$ years. When asked whether human teeth should be used as educational resources, the majority $(89.4 \%)$ answered yes, and concerning the acquisition of psychomotor skills by students, $68.4 \%$ believe that human teeth are more advantageous. He mentioned the disadvantage of using aspects related to biosafety $(36.8 \%)$, difficulty in obtaining teeth (21\%), risks of commercialization $(15.7 \%)$, and ethical aspects $(15.7 \%)$. Most dental professors in the evaluated course understand that the use of human teeth in pre-clinical activities has greater pedagogical potential, in addition to favoring the development of motor skills compared to the use of artificial teeth.

Descriptors: Education, Dental. Tooth. Faculty, Dental.

\section{REFERÊNCIAS}

1. Brasil. Resolução CNE/CES 3/2002. Diário Oficial da União, Brasília/DF, 4 de março de 2002. Seção 1, p. 10. [Acesso em 30 de outubro de 2019]. Disponível em: http://portal.mec.gov.br/cne/arquivos/pdf/C ES032002.pdf

2. Brasil. Parecer CNE/CES 803/2018 [aguardando homologação]. Aprovado em 5 de dezembro de 2018. [Acesso em 25 de maio de 2020]. Disponível em: http://portal.mec.gov.br/index.php?option= com_docman\&view=download\&alias $=127$ 951-pces803-18-1\&category_slug=outubro2019\&Itemid $=30192$

3. Hauser AM, Bowen DM. Primer on preclinical instruction and evaluation. J Dent Educ. 2009;73(3):390-8.

4. Sonntag D, Barwald R, Hulsmann M, Stachniss V. Pre-clinical endodontics: a 
survey amongst German dental schools. Int Endod J. 2008;41:863-8.

5. Nassri MR, Carlik J, da Silva CR, Okagawa RE, Lin S. Critical analysis of artificial teeth for endodontic teaching. J Appl Oral Sci. 2008;16:43-9.

6. Luz DS, Ourique FS, Scarparo RK, et al. Preparation time and perceptions of Brazilian specialists and dental students regarding simulated root canals for endodontic teaching: a preliminary study. J Dent Educ. 2015;79:56-63.

7. Kumar M, Sequeira P S, Peter S, Bhat G K. Sterilisation of extracted human teeth for educational use. Indian J Med Microbiol [serial online] 2005;23:256-58.

8. Tchorz JP, Brandl M, Ganter PA, Karygianni L, Polydorou O, Vach K et al. Pre-clinical endodontic training with artificial instead of extracted human teeth: does the type of exercise have an influence on clinical endodontic outcomes? Int Endod J. 2015;48:888-93.

9. Holden ACL, Dracopoulos SA. Owning the tooth: exploring the ethical and legal issues relating to the use of extracted human teeth in dental education in Australia. Austr Dent J. 2017;62:146-51.

10. Felipe EF, Costa GBM, Júnior NJ, Costa JA. Aspectos éticos da obtenção de dentes por estudantes de uma graduação em Odontologia. Rev Bioét. 2014;22(1):171-5.

11. Medeiros MCS, Costa ICC, Silva EM, Silva LCA, Santos DA, Paiva DFF. Conhecimento de docentes e discentes de um curso de Odontologia sobre os aspectos legais que envolvem a utilização de dentes humanos extraídos. Rev ABENO. 2020;20(1):13-25.

12. Al-Sudani DI, Basudan SO. Students' perceptions of pre-clinical endodontic training with artificial teeth compared to extracted human teeth. Eur J Dent Educ. 2017;21:e72-e75.

13. Bitter K, Gruner D, Wolf O, Schwendicke F. Artificial Versus Natural Teeth for Preclinical Endodontic Training: a Randomized Controlled Trial. Int Endod J. 2016;43:1212-7.

14. Bardin L. Análise de conteúdo. Lisboa: Edições 70; 2000.

15. Decurcio DA, Lim E, Chaves GS, Nagendrababu V,Estrela C, Rossi-Fedele G. Pre-clinical endodontic education outcomes between artificial versus extracted natural teeth: a systematic review. Int Endod J, 2019;52:1153-61.

16. Suebnukarn S, Haddawy P, Rhienmora P, Gajananan K. Haptic Virtual Reality for Skill Acquisition in Endodontics. J Endod. 2010;36(1):53-5.

17. Vincent M, Joseph D, Amory C, Paoli N, Ambrosini P, Mortier E et al. Contribution of Haptic Simulation to Analogic Training Environment in Restorative Dentistry. J Dent Educ. 2020;84(3):367-76.

18. Hashemipour MA, Mozafarinia R, Mirzadeh A, Aramon M, Nassab SAHG. Knowledge, attitudes, and performance of dental students in relation to sterilization/disinfection methods of extracted human teeth. Dent Res J (Isfahan). 2013;10(4):482-8.

19. Morsczeck C, Reichert TE. Dental stem cells in tooth regeneration and repair in the future. Expert Opin Biol Ther. 2018(2):187-96.

\section{Correspondência para:}

Leandro Brambilla Martorell e-mail: 1bmartorell@gmail.com Av. Universitária - Cidade Universitária Centro Universitário de Anápolis UniEVANGÉLICA - Bloco "C" 75075-010 Anápolis/GO 